\title{
Statistics of fine dispersion structures events in energetic particle spectra: their origin and role in the outer magnetosphere
}

\author{
V. N. Lutsenko, E. A. Gavrilova, and T. V. Grechko \\ Space Research Institute RAS, Profsoyuznaya 84/32, Moscow, Russia \\ Received: 30 August 2007 - Revised: 13 May 2008 - Accepted: 20 June 2008 - Published: 31 July 2008
}

\begin{abstract}
Fine Dispersion Structures (FDS) in energetic particle spectra in outer magnetosphere were discovered in DOK-2 experiments onboard of Interball-1 and -2 spacecrafts (1995-2000). First results of these studies were published in 2000-2005. It was shown that FDS can be a result of a gradient-curvature drift of ions and electrons around the Earth after their pulse injection from the geotail plasma sheet to some point in the night side of the outer magnetosphere. Since that time hundreds of such events have been examined additionally. They proved to be a rather common phenomenon in the outer Earth's magnetosphere. We present here statistics of FDS observations and properties which confirms our interpretation and clarifies the role of magnetotail plasma sheet and FDS particles in dynamics and replenishment of the outer radiation belt population.
\end{abstract}

Keywords. Magnetospheric physics (Auroral phenomena; Energetic particles, trapped; Magnetospheric configuration and dynamics)

\section{Introduction}

The discovery of Fine Dispersion Structures (FDS) was possible due to high energy and time resolutions of the DOK-2 instruments onboard of Interball-1 and -2 spacecrafts (19952000) (see Lutsenko et al., 1998). The first results of FDS study were published elsewhere (Lutsenko et al., 2000, 2002a, 2002b, 2005; Kudela et al., 2002). Two types of FDS were observed: 1 ) short duration ( $<5 \mathrm{~min})$ events and 2$)$ long duration $(>5 \mathrm{~min}$ ) events. Examples of spectrograms and single spectra for the second type FDS events can be found in Lutsenko et al. (2000, 2002a, 2005). Some more examples of short and long dispersion events are given in Fig. 1. During the Interball mission about 1000 FDS of both types were

(vlutsenk@iki.rssi.ru)

observed. Earlier we have focused mainly on long duration events and more than 600 of them were analyzed in detail. Dependences of these FDS occurrence on spacecrafts positions (R, L, MLT, MLAT values), on magnetosphere and solar wind parameters $\left(K_{p}\right.$ and $D_{s t}$ indexes, $P_{S W}, V_{S W}, B_{Y}$ and $B_{Z}$ IMF values) so as the MLT dependence of FDS duration and $d E / d t$ at fixed proton and electron energies were studied. For a part of FDS events trajectories of particles were found using a simulation of their motion in model magnetic fields (Lutsenko et al., 2005). We give here a statistical survey of FDS events properties which allows making conclusions on their nature and their role in the outer magnetosphere dynamics.

\section{Two types of FDS events and their analysis method}

We used a simple but very effective method of FDS analysis based on high energy and time resolutions of DOK-2 instrument. Two types of dispersion were considered:

1. Time-of-flight type dispersion. After a pulse particles generation at $t=t 0$, at the distance $s$ from the observer he we will observe at some moment $t$ particles with the velocity $v$ and the energy $E$. Here $v=\frac{s}{(t-t 0)}$ and $E=\frac{m v^{2}}{2}=\frac{m s^{2}}{2 \cdot(t-t 0)^{2}}$, where $m$ is a mass (for nonrelativistic ions).

We should observe a linear time dependence of $v^{-1}$ :

$$
\frac{1}{v}=\frac{1}{s} \cdot(t-t 0)
$$

The time dependence of $E^{-1}$ will be nonlinear here:

$$
\frac{1}{E}=\frac{2}{m \cdot s^{2}}(t-t 0)^{2} .
$$

Published by Copernicus Publications on behalf of the European Geosciences Union. 

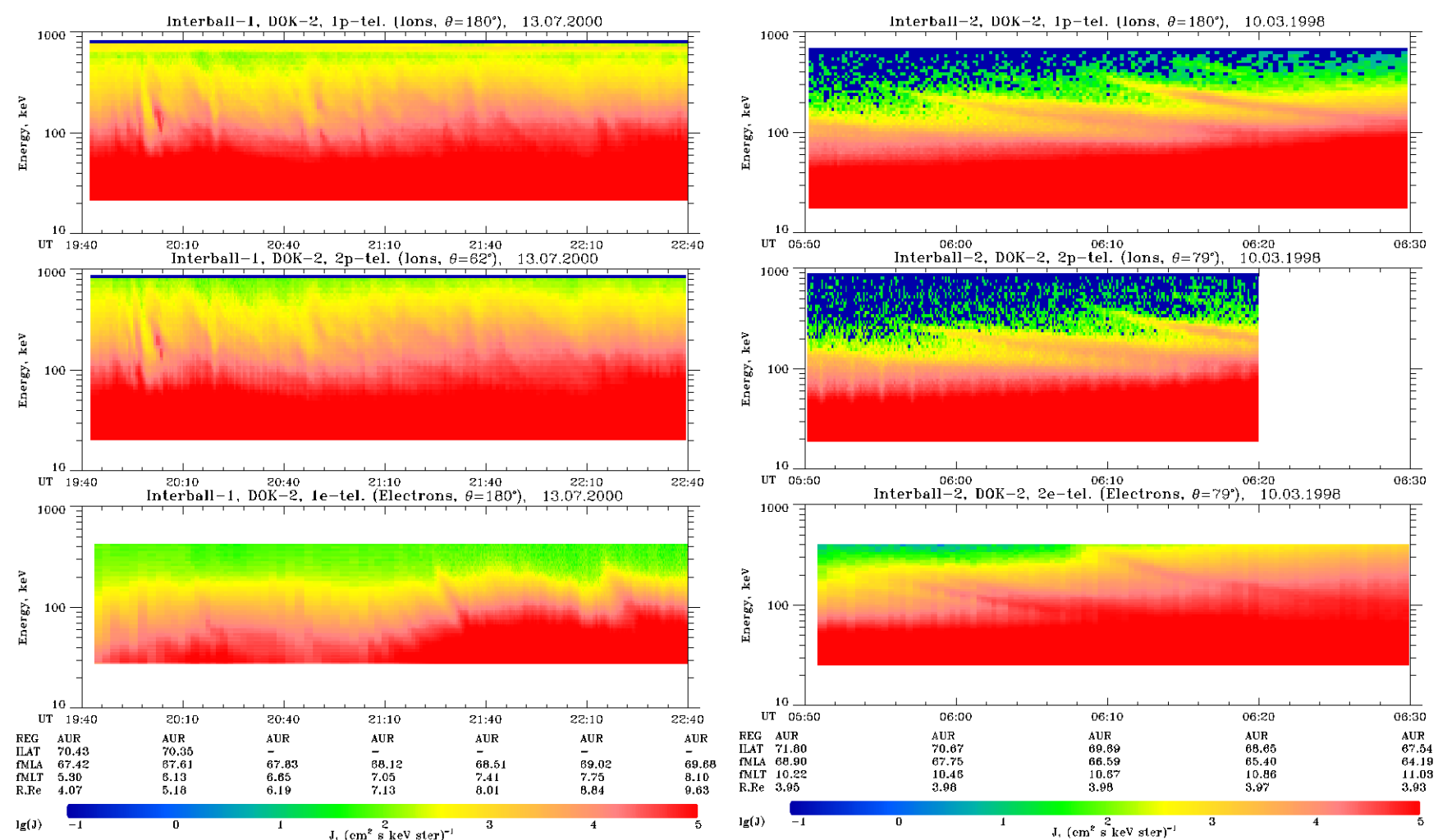

Fig. 1. Example of two types of FDS events. Left spectrograms (Interball-1): many short FDS events in the night auroral region on 13 July 2000 at $R=4-9 R_{E}$. Right spectrograms (Interball-2): several long FDS in the day side auroral region on 10 March 1998. Two upper spectrograms correspond to $1 \mathrm{p}$ and $2 \mathrm{p}$ ion telescopes, the bottom ones to $1 \mathrm{e}$ (left) and $2 \mathrm{e}$ (right) electron telescopes.

At any moment we will observe protons with energy $E_{p}$ and $\alpha$-particles with the same velocity and energy $E_{\alpha}=4 \cdot E_{p}$.

2. Gradient drift type dispersion. After a pulse injection into a small volume of outer magnetosphere (a closed magnetic field line region at $L=6-16 R_{E}$ ) particles start a gradient-curvature drift around the Earth. As the trajectory shape here is very complicated and the bounce period is rather short $(\sim 1 \mathrm{~s}$ for electrons and $\sim 20 \mathrm{~s}$ for proton with $E=300 \mathrm{keV}$ at $L=6$ ) it is more convenient to examine an azimuthal position $\varphi$ or a magnetic local time $\mathrm{MLT}_{F}$ of a field line foot corresponding to the particle position.

An angular drift velocity $v_{\varphi}=d \varphi / d t \sim E / q$ (see e.g. Roederer, 1970) and does not depend on the particle mass. So in this case $E / q=k \cdot \Delta \varphi /(t-t 0)$ and we will have a linear time dependence of $E^{-1}$ :

$\frac{q}{E}=\frac{(t-t 0)}{k \cdot \Delta \varphi}$.

Here $k$ is a constant which depends on the location of the drift shell, $\Delta \varphi=\varphi-\varphi 0$ - the azimuthal distance be- tween injection and observation points. For relativistic electrons we must use the quantity $E^{*}$ instead of $E$ :

$$
E^{*}=E \cdot \frac{\left(E+2 \cdot m_{0} c^{2}\right)}{2 \cdot\left(E+m_{0} c^{2}\right)} .
$$

For $v^{-1}$ the time dependence is nonlinear now:

$$
v^{-1}=\sqrt{\frac{m}{2 q \cdot k \cdot \Delta \varphi}} \cdot \sqrt{(t-t 0)} .
$$

By the gradient drift the angular drift velocity $v_{\varphi}$ is proportional to $E / q$ and as at any moment we will observe ions with the same velocity $v_{\varphi}$ alpha-particles will have the energy $E_{\alpha}=2 \cdot E_{p}$.

We observed both types of dispersion in DOK-2 experiment. Examples of $1 / E$ and $1 / v$ analysis for protons in several short and long FDS events are given in Fig. 2. For each particular spectrum we measure an energy $E$ corresponding to the FDS line maximum and a time $t$ of the spectrum accumulation interval middle. Points on these plots give the time dependence of quantities proportional to $1 / E$ and $1 / v$. Then we make least square fittings of points in both Y-scales. Experimental points that lay better on a straight line in $1 / E$ 

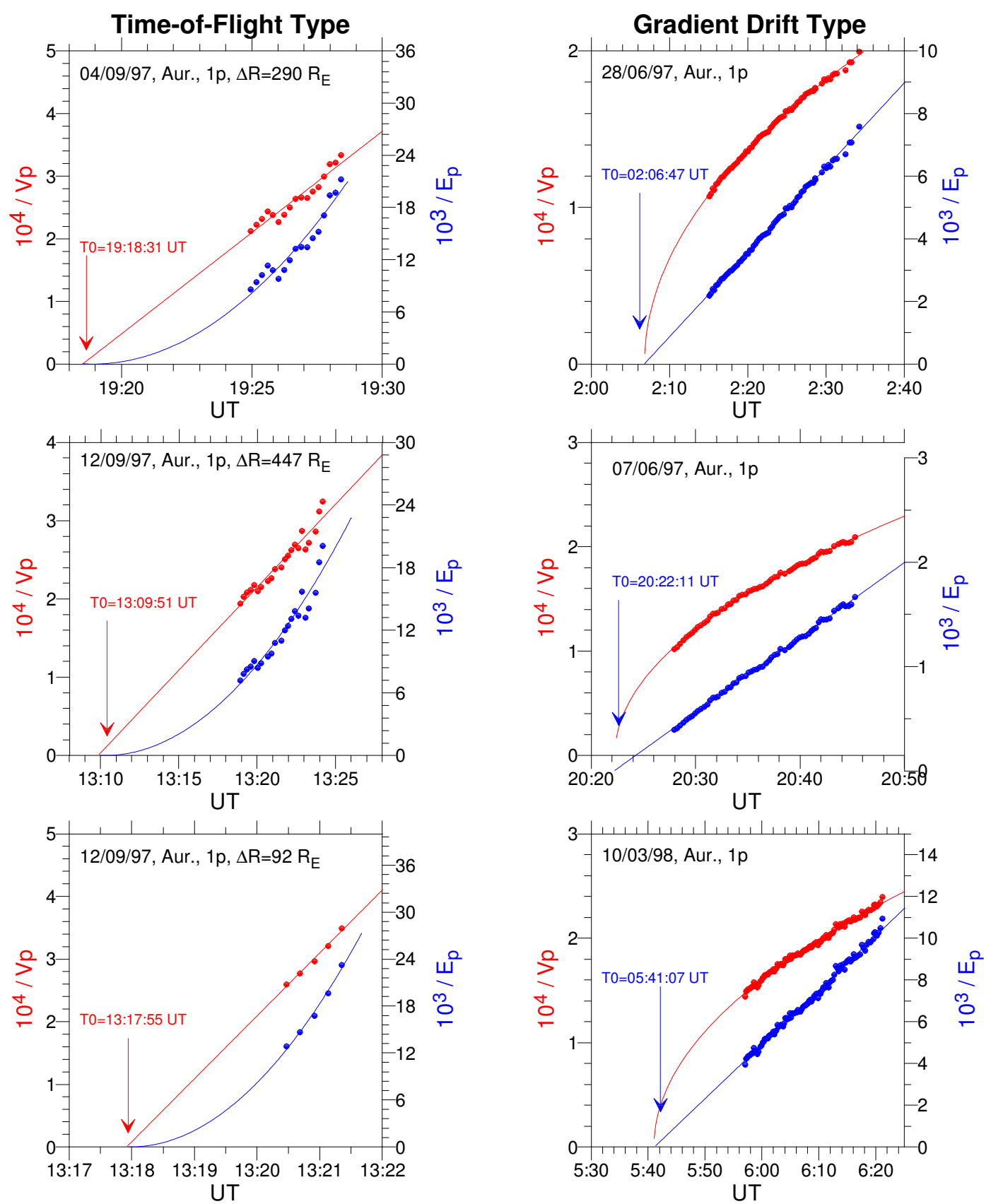

Fig. 2. Examples of $1 / E$ (blue) and $1 / v$ (red) analysis for protons in several FDS events of both types.

Y-scale correspond to gradient drift type of dispersion, if it is fulfilled for $1 / v$ scale - correspond to "time-of-flight" type. In the first case points in $1 / v$ Y-scale were best fitted by $Y=A 1 \cdot \sqrt{(T-T 0)}$ function. In the second case points in $1 / E$-scale occurred best fitted by $Y=A 2 \cdot(T-T 0)^{2}$ function. Here $A 1$ and $A 2$ are constants. In both cases precise values of $T 0$ can be obtained from the straight line fitting expression.

Therefore least square fits of experimental points by a straight line allows to:
- find the dispersion type,

- find the time $T 0$ of the drift motion start (injection time),

- estimate the distance to the source position from the slope of the line,

- identify ion species (protons and alpha-particles, see Fig. 3), and 

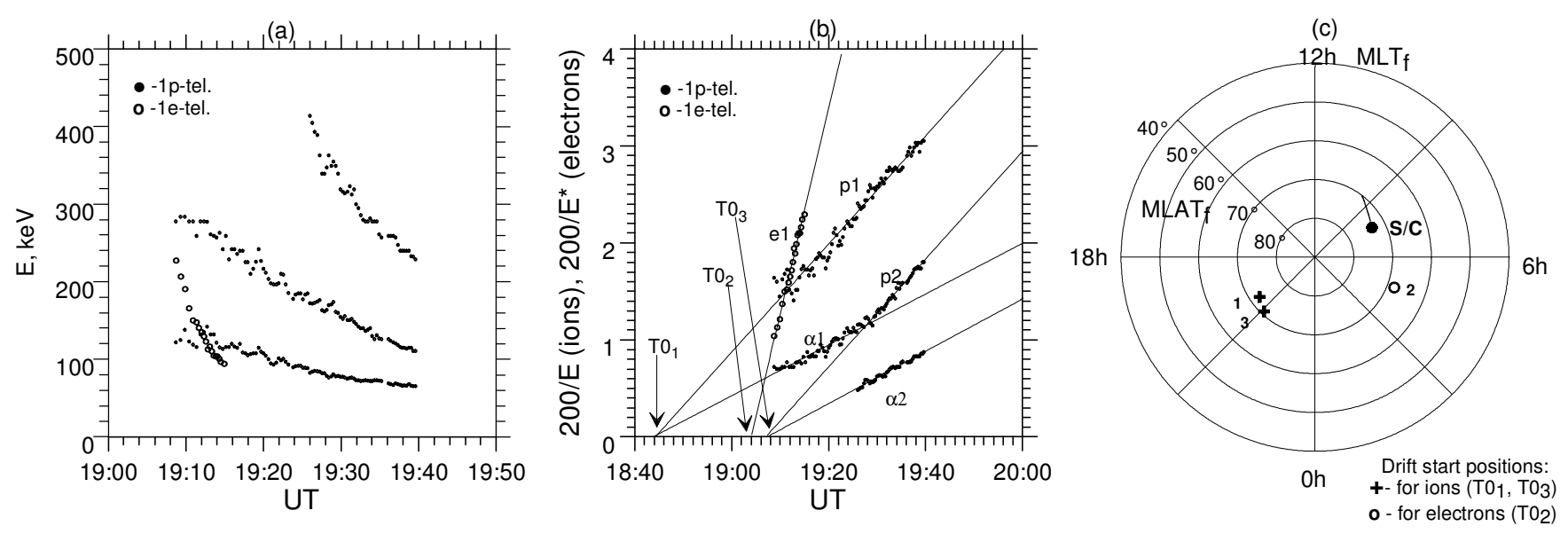

Fig. 3. Example of the analysis for multiple injection event on 14 September 1996. Three ion and one electron FDS (a) correspond to 2 injections for ions and 1 for electrons (b). The right plot (c) shows the calculated injection positions and the Interball-2 position change during FDS event (black point - at the FDS start).

- find FDS events corresponding to particles which have made one and more full turns around the Earth (two or more FDS for particles of the same sort have the same $T 0$ value). In this case we can determine the drift period as a function of the particle energy (Lutsenko et al., 2005).

The source position (injection point) $\varphi 0$ can be determined by backtracing the particle motion in some magnetic and electric field models from the observation position $\varphi 1$ and time $T 1$ to the time $T 0$. Usually we have used a combination T96+IGRF for the magnetic field and a Volland (Volland, 1978) for the electric field models. Figure 3 illustrates results of such analysis for one multiple injection event (Lutsenko et al., 2002b) on 14 September 1996 with three FDS for ions and one for electrons (Fig. 3a). The $1 / E$ analysis (Fig. 3b) reveals 3 particle injections with $T 0_{1}=18: 44: 03$ UT, $T 0_{2}=19: 07: 09$ UT (ions) and $T 0_{3}=19: 04: 00$ UT (electrons). The analysis allowed to identify ion species (protons and $\alpha$ particles). The spacecraft and calculated source positions are shown in Fig. 3c.

Interball data show that the average occurrence frequency of short, "time-of-flight" type FDS events in the night side magnetosphere and in the near plasma sheet is $8-10$ per hour by observation at one point. The total frequency of such events in the whole night side must be much greater. It is natural to assume that short FDS are produced by the direct arrival of particles from the geotail plasma sheet and their reflection back from the outer magnetosphere. This conjecture was confirmed by simulation of proton motion from observation points for several such events. Here and further under the outer magnetosphere we have in a view a close field line region with L-parameters 6-16 $R_{E}$.

Figure 4 shows results of $150 \mathrm{keV}$ proton motion simulations for two FDS events in the night side auroral region on 4 September 1997 starting at 19:26 UT and 20:29 UT (Interball-2). The first (No 5) was of "time-of-flight" type, while the second one (No 6) - of gradient drift type. For the No 5 event a backtracing simulation from the observation position leads to a distant plasma sheet acceleration position. The forward simulation shows that the protons arriving from the plasma sheet were reflected shortly after passing the spacecraft and returned back to the plasma sheet. For the event No 6 we have a normal gradient-curvature drift around the Earth. Two more examples of proton tracing for short FDS (also with $E=150 \mathrm{keV}$ ) are given in Fig. 5. Here positions of Interball-2 are marked by black points and proton motion directions are shown by arrows. As for No 5 event in Fig. 4 the protons were coming from a distant plasma sheet and then reflected back from the magnetosphere. Certainly the T96+IGRF combination of magnetic field models used here can hardly be applicable to such a distant and unstable plasma sheet region $\left(X_{S M}<-20 R_{E}\right)$ and we can find only very rough estimates of particle acceleration positions.

The FDS particle acceleration can occur in the magnetotail neutral sheet by pulses of an inductive electric field caused by spontaneous current filaments disruptions followed by a local magnetic field reconnection and impulsive increase of $B_{Z}$ magnetic field component (see details in Sect. 9).

The frequency of longer, drift type events is much lower. From August 1995 to October 2000311 electron and 299 ion distinct FDS events of this type were observed in the auroral zones during $4811 \mathrm{~h}$ of observations by DOK- 2 on Interball-1 and -2 spacecrafts. It gives an average frequency 0.13 per hour, which is at least 70 times lower than for "timeof-flight" type. 

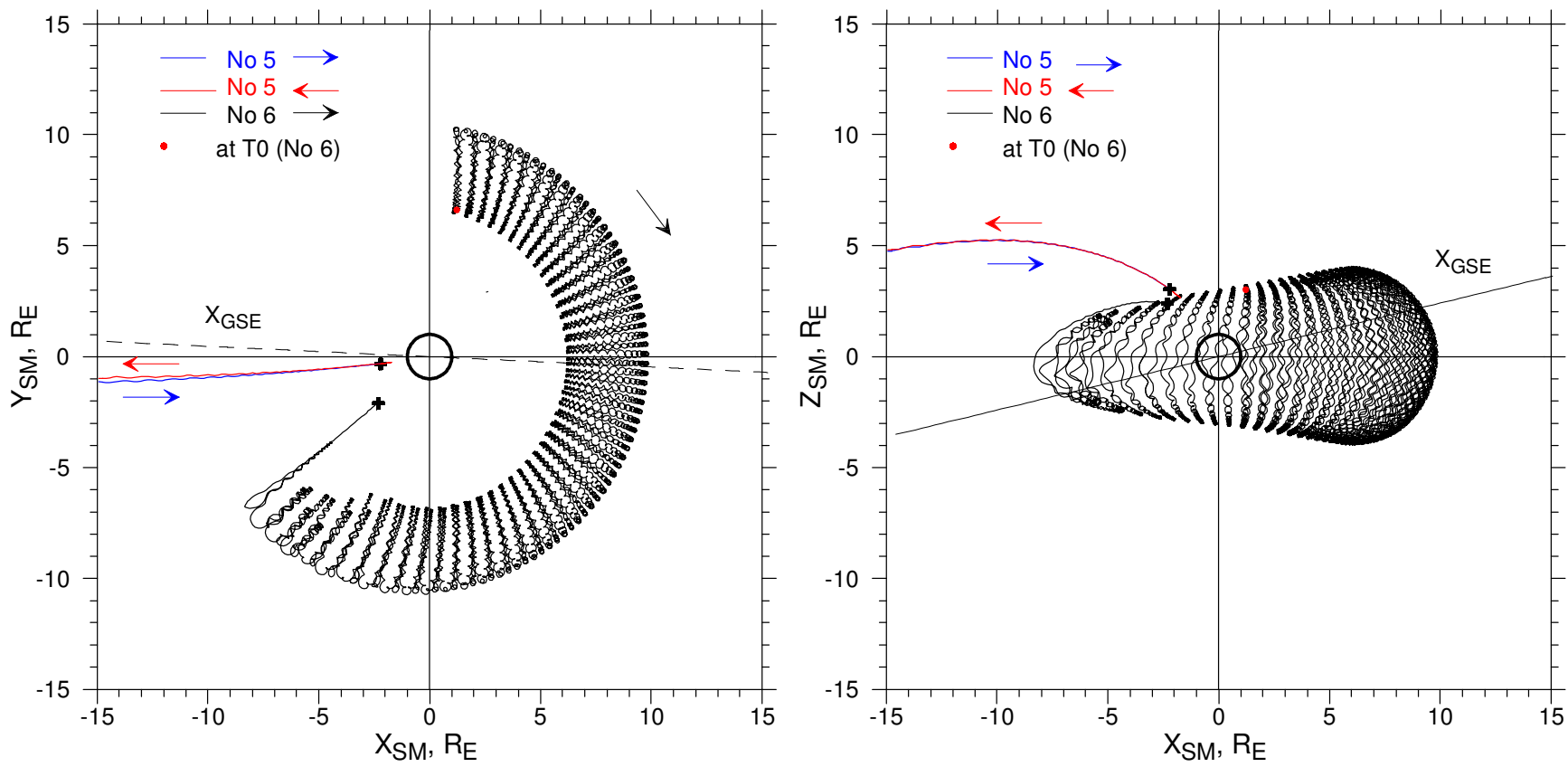

Fig. 4. Simulated $150 \mathrm{keV}$ proton trajectories for two FDS events on 4 September 1997: No 5 of "time-of-flight" type and No 6 of gradient drift type. Interball-2 observation positions are marked by black crosses, directions of particles motion by arrows. The drift start position for No 6 is marked by red points.
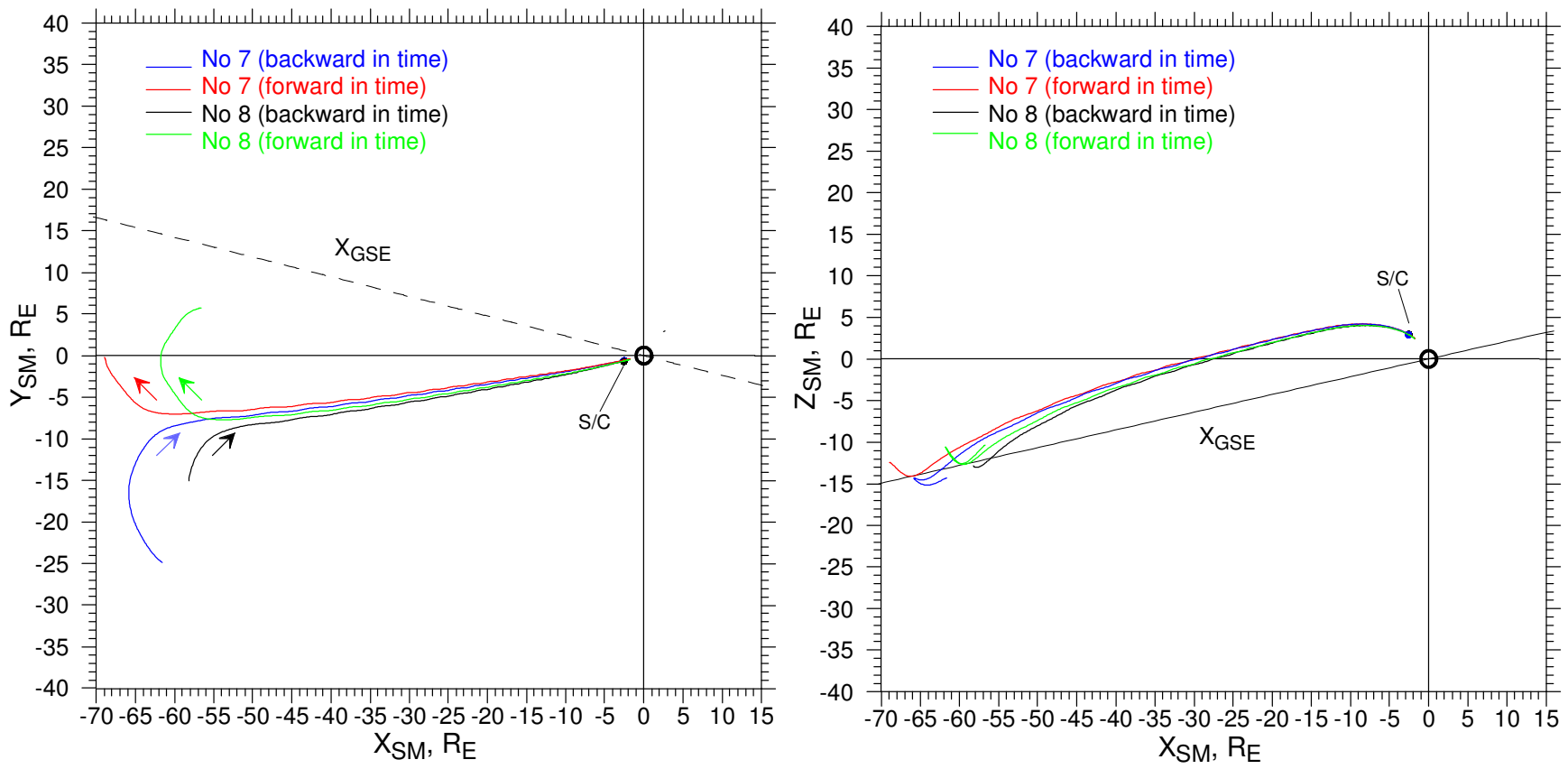

Fig. 5. Results of $150 \mathrm{keV}$ proton motion simulation for two short FDS events on 12 September 1997 at 18:53:15 and 18:58:40 UT. Interball-2 observation positions are marked by black points, directions of particles motion by arrows.

\section{Statistics of FDS observation positions}

Under a single FDS event we will imply 1 or 2 (protons and alpha-particles) distinct fine dispersion structures in spec- trograms having unique $T 0$ value. In distributions below we used all data on gradient drift FDS events for both Interball spacecrafts excluding events with a few spectra and those which were observed only after one full turn around 

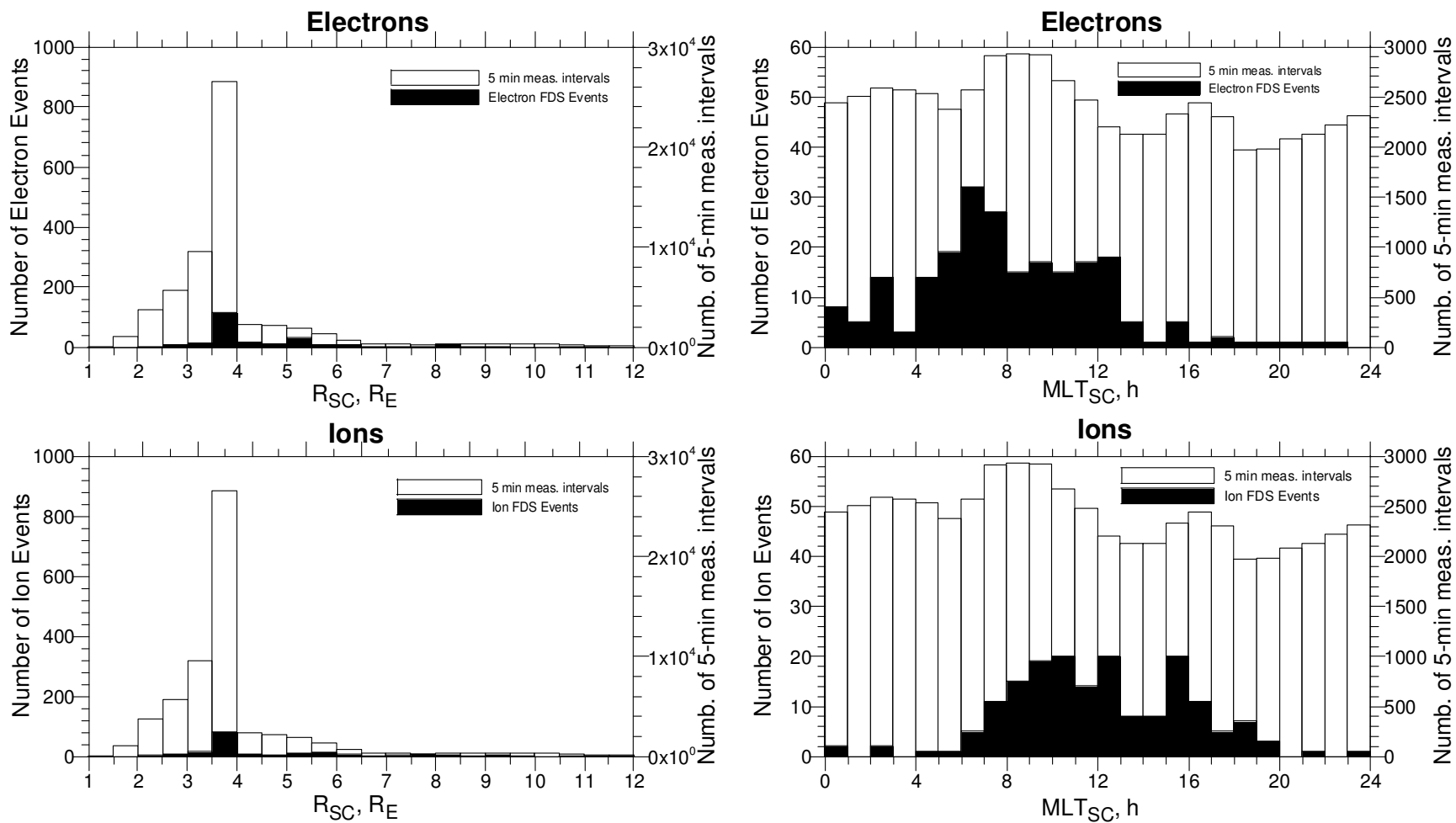

Fig. 6. The $R_{S C}$ (left) and MLT $S C$ (right) dependence of the FDS events numbers.

the Earth. We studied dependence of electron and ion FDS events numbers on spacecrafts position: $R_{S C}, \mathrm{MLT}_{S C}$ (Fig. 6) and on magnetic field line foot latitude $\mathrm{MLAT}_{F}$ and McIlwain parameter $L$ (Fig. 7). All distributions of FDS events numbers (black histograms) are accompanied by distributions of 5-min measurements intervals numbers in auroral zones (white histograms). We present two separate histograms instead of one for FDS events numbers per unit of measurement time. This kind of normalization allows to estimate easily the statistical accuracy of the results for black histograms and relative measurements duration for different parameter values.

It can be seen that maximum number of FDS events were observed near $R_{S C}=3.5-4 R_{E}$. The distribution in MLT $_{S C}$ is rather broad: $0-16 \mathrm{~h}$ for electrons and $6-20 \mathrm{~h}$ for ions. This difference may be due to the difference in drift directions and in drift start positions (see Sect. 6). The histograms in Fig. 7 give drift shells distribution of FDS events numbers. FDS structures for both types of particles were observed at $L=6$ 16 with maximum at $L=10$. Therefore FDS events can hardly be observed from spacecrafts on geostationary orbits.

\section{Dependence of FDS numbers on magnetosphere and solar wind states}

While the period of Interball work was characterized by rather quiet magnetosphere and solar wind states we checked the dependence of FDS events numbers on $D_{s t}$ and $K_{p}$ indexes (magnetosphere), $P_{S W}, V_{S W}$ (solar wind) and $B_{Y}$ and $B_{Z}$ (interplanetary magnetic field). Figures 8-10 do not show any preference of disturbed states. So the FDS particles generation occurs essentially at quiet magnetosphere and solar wind states and most likely have no relations to magnetic storms, substorms and other great scale disturbances. Values of statistical parameters for FDS event numbers distributions shown in Figs. 6-10 are given in the Table 1. They are in agreement with conclusions made above after visual figures inspection.

\section{Dependence of the FDS duration and $d E / d t$ at a fixed energy on the spacecraft MLT $_{F}$}

It follows from the low width of the FDS lines in spectra that the duration of an injection process and the longitude dimension of an injection region must be small (see Sect. 9 and Lutsenko et al., 2005). The simplest estimate of the distance from the observation position to the drift start place can be made from the dispersion structure duration: the smaller is 

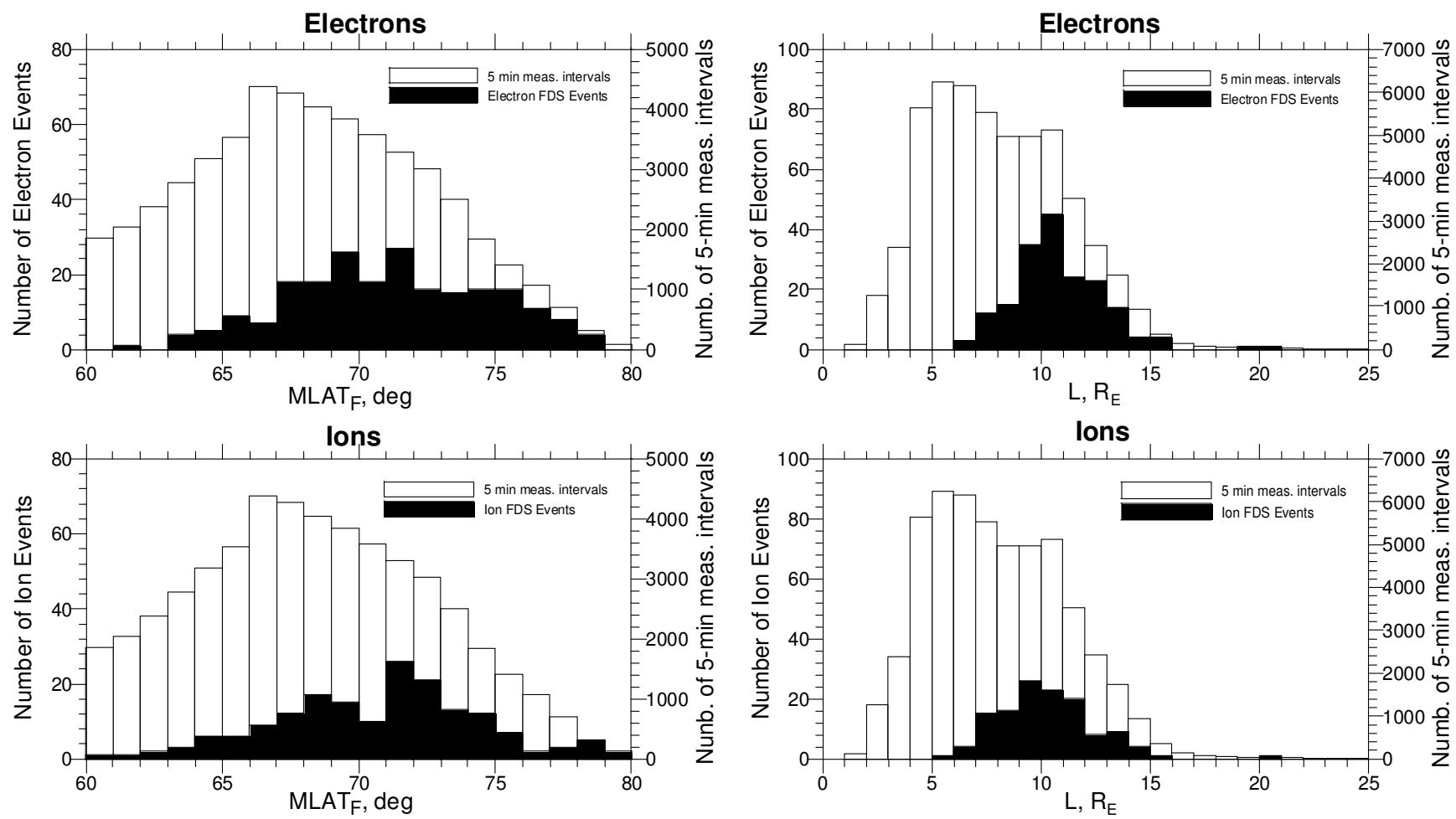

Fig. 7. The $\operatorname{MLAT}_{F}$ (left) and $L$ (right) dependence of the FDS events numbers.

Table 1. Statistical parameters of FDS event numbers distributions for $R_{S C}, \operatorname{MLT}_{S C}, \operatorname{MLAT}_{F}, L, D_{s t}, K_{p}, P_{S W}, V_{S W}, B_{Y}, B_{Z}$.

\begin{tabular}{llccccccc}
\hline Variable & Ions/Electrons & Events Numb. & Min val. & Max val. & Mean & Median & Stand. deviation & Stand. error \\
\hline$R_{S C}, R_{E}$ & ions & 179 & 2.35 & 12.73 & 5.011 & 3.98 & 2.177 & 0.163 \\
$R_{S C}, R_{E}$ & electrons & 223 & 2.44 & 11.02 & 4.521 & 3.99 & 1.390 & 0.093 \\
$\mathrm{MLT}_{S C}, \mathrm{~h}$ & ions & 179 & 0.02 & 23.95 & 12.163 & 12.0 & 0.300 & 4.015 \\
$\mathrm{MLT}_{S C}, \mathrm{~h}$ & electrons & 223 & 0.29 & 22.77 & 8.146 & 7.52 & 4.108 & 0.275 \\
$\mathrm{MLAT}_{F}, \mathrm{deg}$ & ions & 174 & 60.24 & 81.29 & 70.794 & 71.18 & 3.858 & 0.292 \\
$\mathrm{MLAT}_{F}, \mathrm{deg}$ & electrons & 221 & 61.8 & 80.3 & 71.238 & 71.17 & 3.698 & 0.249 \\
$L, R_{E}$ & ions & 128 & 5.94 & 20.12 & 10.267 & 10.095 & 2.203 & 0.195 \\
$L, R_{E}$ & electrons & 181 & 6.44 & 20.12 & 10.797 & 10.47 & 2.11 & 0.157 \\
$D_{s t}, \mathrm{nT}$ & ions & 179 & -51 & 14 & -14.1 & -13 & 12.2 & 0.911 \\
$D_{s t}, \mathrm{nT}$ & electrons & 223 & -56 & 14 & -13 & -12 & 13.4 & 0.900 \\
$K_{p}$ & ions & 179 & 3 & 53 & 18.7 & 17 & 11.4 & 0.853 \\
$K_{p}$ & electrons & 223 & 0 & 57 & 16.4 & 13 & 11.5 & 0.770 \\
$P_{S W}, \mathrm{nP}$ & ions & 169 & 0.6 & 6.7 & 2.07 & 1.8 & 1.11 & 0.085 \\
$P_{S W}, \mathrm{nP}$ & electrons & 192 & 0.6 & 11.4 & 2.09 & 1.9 & 1.455 \\
$V_{S W}, \mathrm{~km} / \mathrm{s}$ & ions & 169 & 282 & 616 & 398 & 393 & 70.26 & 0.105 \\
$V_{S W}, \mathrm{~km} / \mathrm{s}$ & electrons & 192 & 289 & 622 & 389.6 & 286.5 & 68.08 & 5.405 \\
$B_{Y}, \mathrm{nT}$ & ions & 110 & -7.4 & 5.4 & 0.01 & -0.3 & 2.67 \\
$B_{Y}, \mathrm{nT}$ & electrons & 108 & -9.5 & 17 & 0.479 & 0.75 & 5.28 & 4.913 \\
$B_{Z}, \mathrm{nT}$ & ions & 110 & -7.1 & 3.2 & -0.771 & -0.45 & 1.95 \\
$B_{Z}, \mathrm{nT}$ & electrons & 108 & -8.3 & 11.1 & -0.239 & 0.0 & 2.966 & 0.254 \\
\hline
\end{tabular}



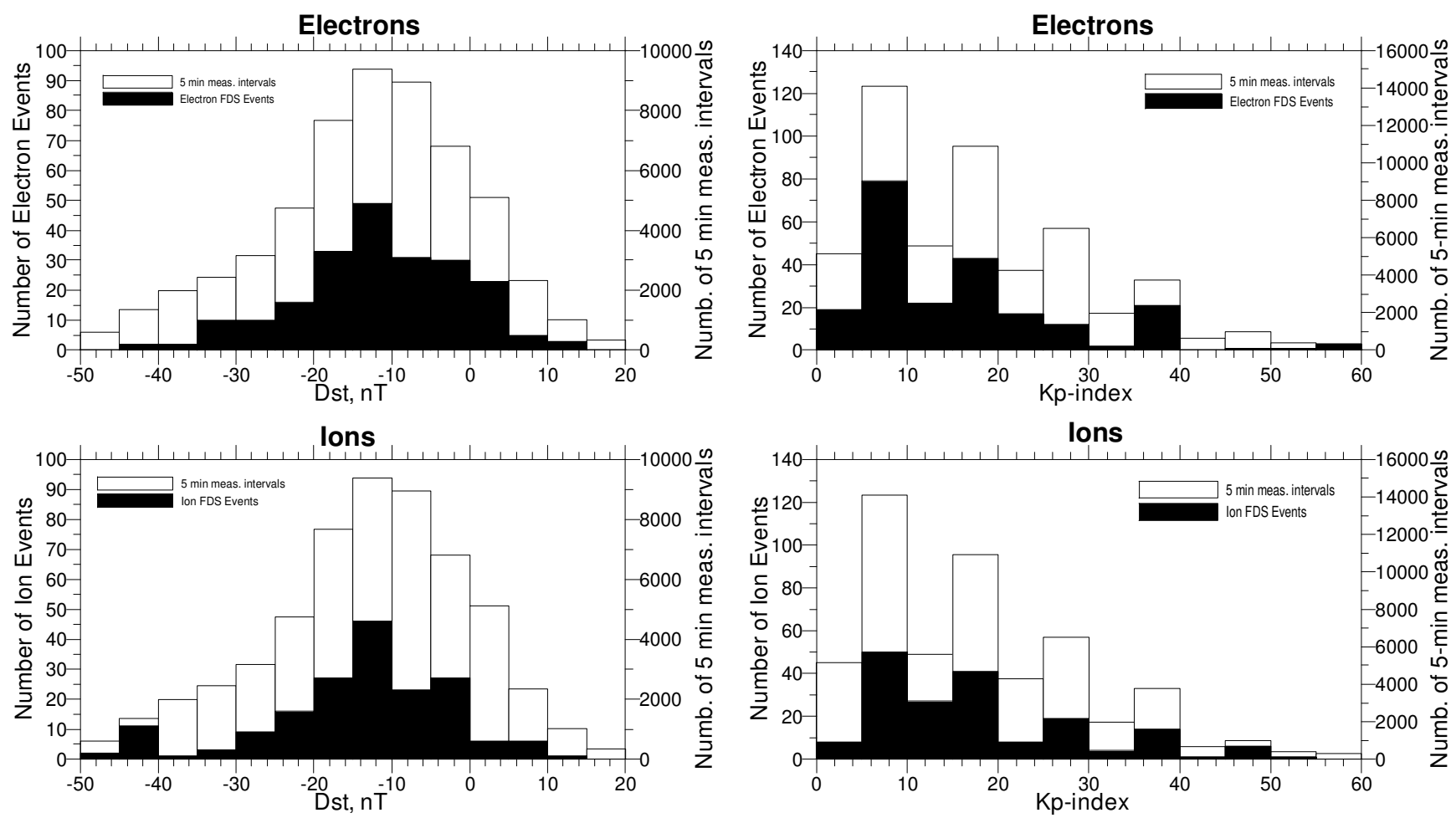

Fig. 8. The $D_{s t}$ (left) and $K_{p}$ (right) parameters dependence of the FDS events numbers.
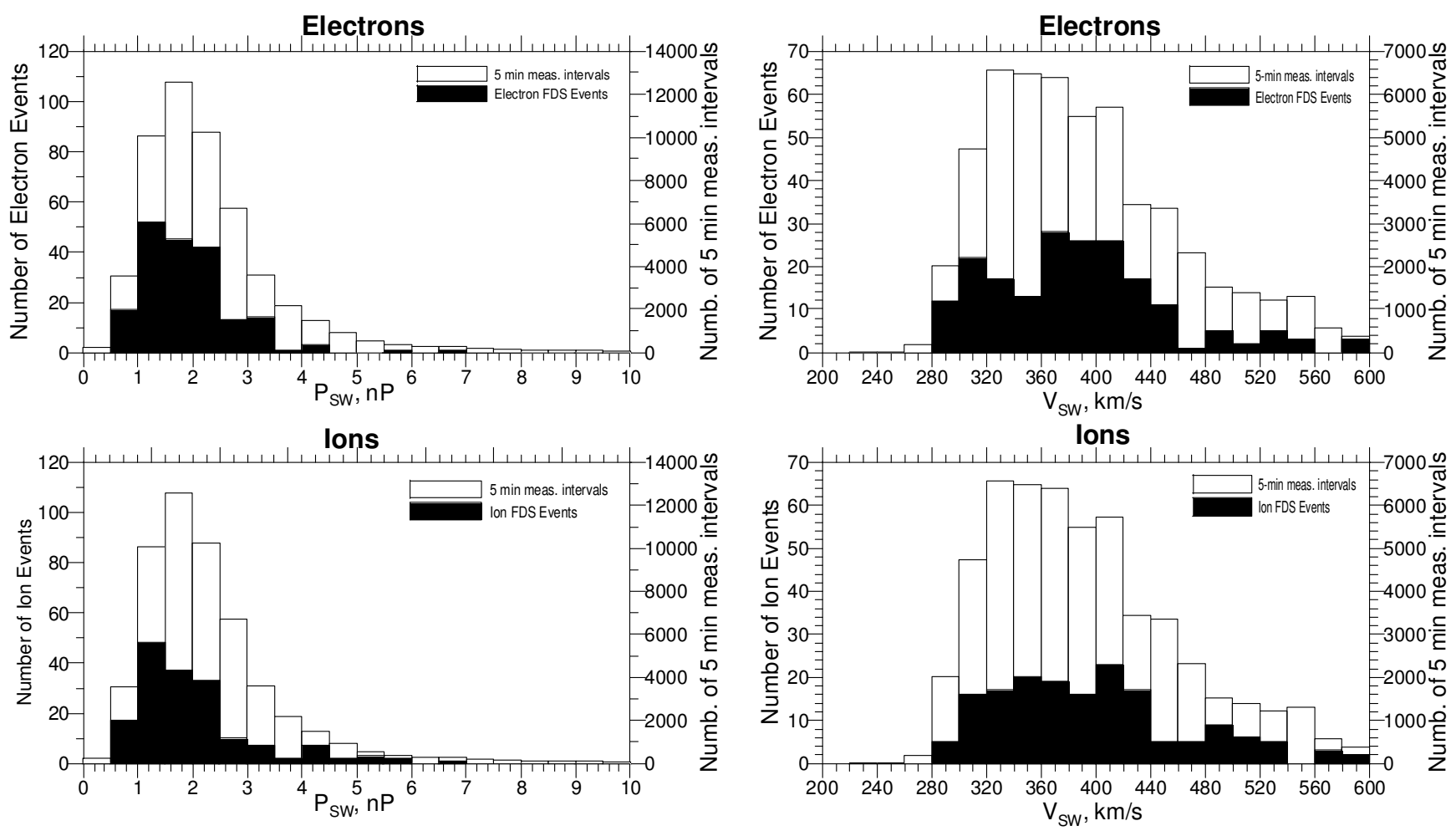

Fig. 9. The $P_{S W}$ (left) and $V_{S W}$ (right) dependence of the FDS events numbers. 

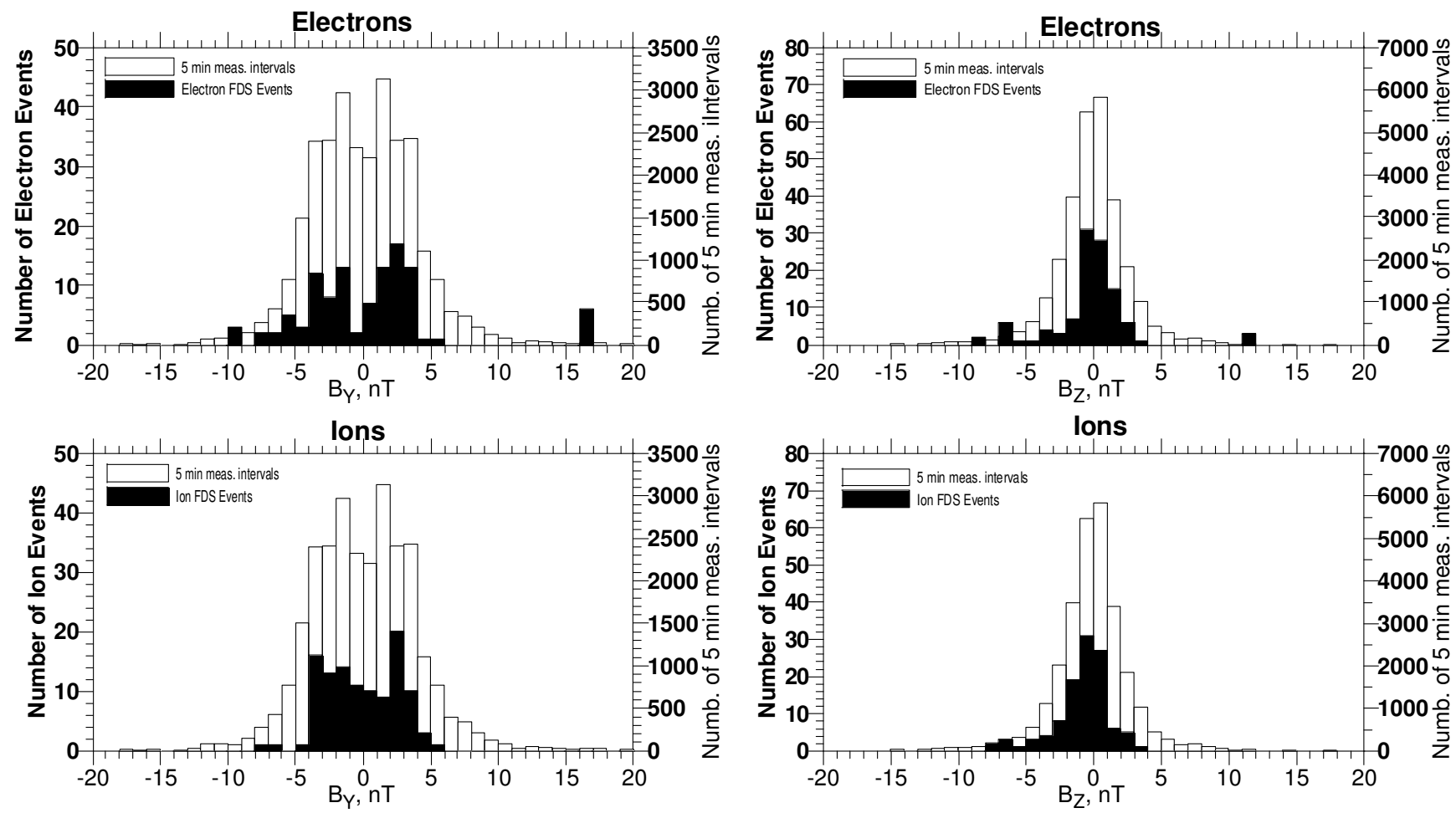

Fig. 10. Dependence of the FDS event numbers on the interplanetary magnetic field components (GSE) $B_{Y}$ (left) and $B_{Z}$ (right).
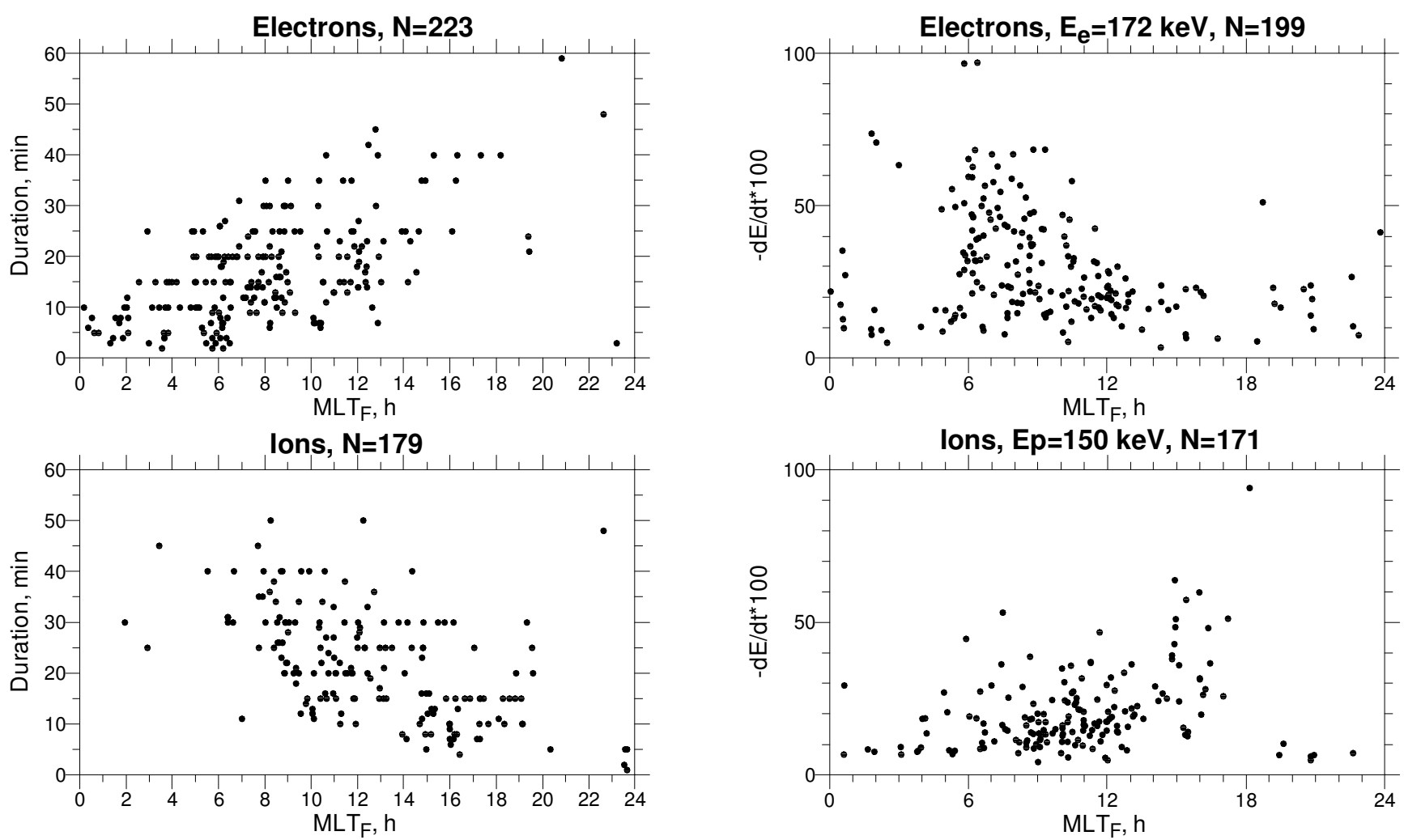

Fig. 11. The $\mathrm{MLT}_{F}$ dependence of FDS duration (left) and $d E / d t$ at a fixed energy (right). 


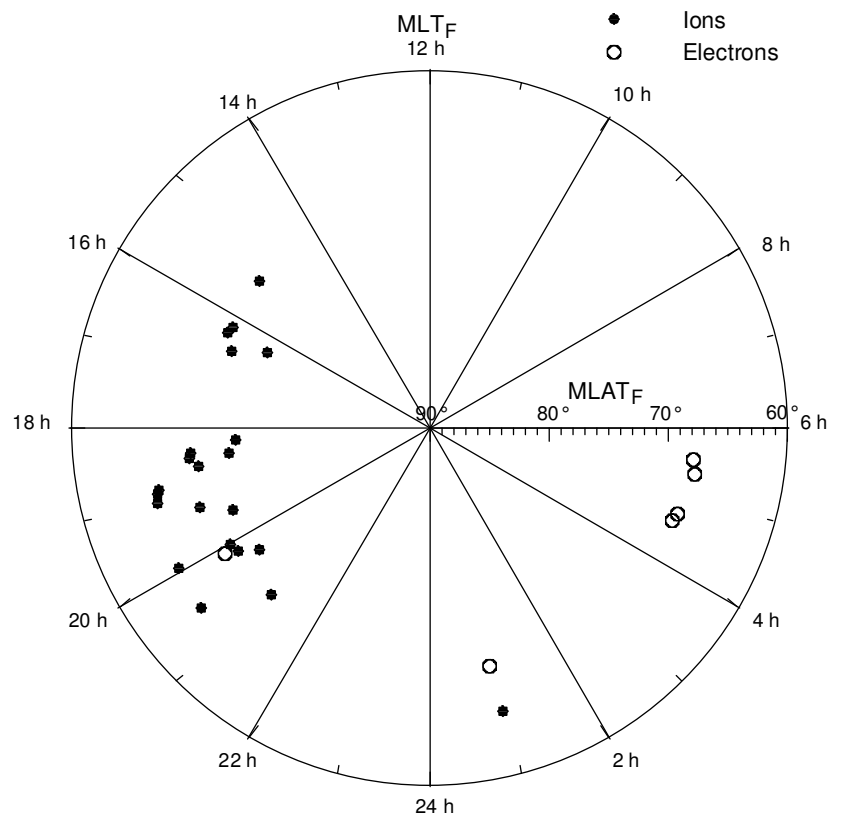

Fig. 12. Injection positions of FDS particles in $\operatorname{MLAT}_{F}-\mathrm{MLT}_{F}$ coordinates for magnetic field line foot.

this distance, the shorter is FDS. The difficulty here is the uncertainty of the start and the end of the FDS line which can be out of measurements time interval, can be masked by other lines or by a background spectrum. This can increase the point spread in corresponding graph. The other possibility is to measure velocity of a peak energy change $d E / d t$ at any fixed $E$ value. It follows from the Eq. (3) that $\frac{d E}{d t}=-\frac{E^{2}}{q \cdot k \cdot \Delta \varphi}$ must smoothly decrease with the increase of the distance from the injection position $\Delta \varphi$. We used both these methods (see Fig. 11).

These distributions confirm on a large statistical base our conclusions made previously after several events analysis:

1. the FDS particles start the drift somewhere in the night side of the outer magnetosphere,

2. ions propagate clockwise, while electrons - anticlockwise (looking from the north), just what we can expect for the gradient drift. The points spread is explained by the spread in drift start positions (see next section).

\section{Injection positions distribution}

In Lutsenko et al. (2005) we found the injection position for several FDS events. It was done using a backward in time simulation of particle motion from the observation time and position to the start time $T 0$ which can be found by the analysis described above. Figure 12 shows these positions for some more events ( 22 for ion and 6 for electrons) in magnetic field line foot coordinates MLAT $_{F}-\mathrm{MLT}_{F}$. We used IGRF and Tsyganenko-96 models for internal and external magnetic fields. While the position distribution has significant spread it is seen that ions were injected mainly at $\mathrm{MLT}_{F}=16$ $21 \mathrm{~h}$, while electrons - at $\mathrm{MLT}_{F}=0-6 \mathrm{~h}$. The difference can be explained by gradient drift of particles during their propagation in the plasma sheet from the distant acceleration point to the injection position (see e.g. Sarafopoulos et al., 2001). This difference is in agreement with dependences of FDS duration and $d E / d t$ on $\mathrm{MLT}_{F}$ shown in Fig. 11.

\section{How long can FDS particles stay in outer magneto- sphere after their injection?}

Our simulations of FDS particle motion using the T96+IGRF magnetic field model showed that even in a stable magnetosphere state part of the particles can come out of the magnetopause or precipitate to the ionosphere. For any given energy and start position it depends on initial pitch angle. For an 24 April 1997 event (Interball-2) we changed this angle in the range of ion telescope angular aperture $\left(12.5^{\circ}\right)$ with a step of $0.5-0.8^{\circ}$ and found that: $62 \%$ of 95 protons $(E p=230$, 275 and $394 \mathrm{keV}$ ) make one full turn, 33\% escape the magnetosphere and 5\% precipitate into the ionosphere.

In DOK-2 data we found 10 events (6 for electrons and 4 for ions) for which the observation time in a given drift shell was sufficiently long and we observed particles making 1-3 full turns around the Earth (see e.g. Fig. 4 in Lutsenko et al., 2005). So being injected to the outer magnetosphere FDS particles can drift here during a rather long time at least 30-60 min depending on the energy and the particle type.

\section{Contribution of FDS particles to a total energy den- sity and a total energy flux of energetic ions and elec- trons in the auroral regions}

At high energies $(E>100 \mathrm{keV})$ intensities in maxima of FDS lines more than 10-30 times exceed that for the background spectrum. But to estimate the contribution of these thin lines to the total energetic particle population it is more correct to find an average spectrum for the whole FDS event and to compare it with the average spectrum before the event (the background). The comparison of these spectra will help to estimate the role of FDS particles in replenishment of the energetic particles population which experience steady losses because of particles escape the outer magnetosphere and precipitation to the ionosphere. We have made such calculations for two single FDS events: 28 June 1997 (for ions) and 7 July 1997 (for electrons). In both cases the background spectrum was stable enough. Figure 13 gives ion and electron spectra for these two events. At $E>100 \mathrm{keV}$ intensities in average spectra during these FDS events are 8-10 times greater than those for background spectra, at lower energies the ratio decrease. As to the whole spectrum even a single FDS event gives a significant $(>50 \%)$ addition to the total energy flux 

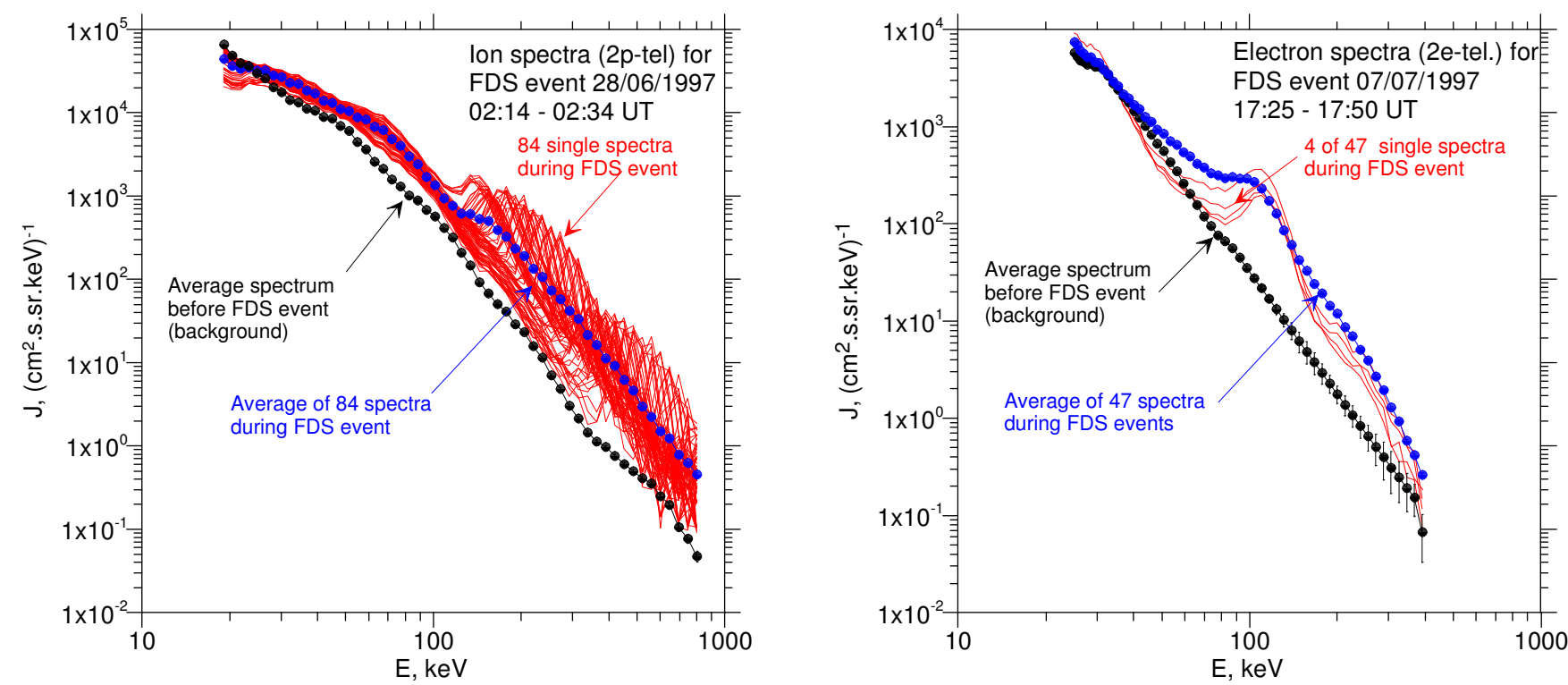

Fig. 13. Average spectra before and during single FDS events for ions (left) and electrons (right). Separate spectra with FDS are shown by thin red lines.

Table 2. Contribution of FDS particles to a total energy density (ED) and a total energy flux (EF) of energetic ions and electrons in auroral zones (see Fig. 13).

\begin{tabular}{lcccc}
\hline Average Spectrum & $\mathrm{ED}_{I}, \mathrm{keV} / \mathrm{cm}^{3}$ & $\mathrm{EF}_{I}, \mathrm{keV} / \mathrm{cm}^{2} / \mathrm{s} / \mathrm{sr}$ & $\mathrm{ED}_{E}, \mathrm{keV} / \mathrm{cm}^{3}$ & $\mathrm{EF}_{E}, \mathrm{keV} / \mathrm{cm}^{2} / \mathrm{s} / \mathrm{sr}$ \\
\hline Before FDS event & 1.3 & $3.10^{7}$ & $2.9 .10^{-3}$ & $2.9 .10^{6}$ \\
During FDS event & 2.0 & $5.10^{7}$ & $4.4 .10^{-3}$ & $4.8 .10^{6}$ \\
Increase in \% & $+54 \%$ & $+67 \%$ & $+52 \%$ & $+66 \%$ \\
\hline
\end{tabular}

(EF) and the total energy density (ED) of ambient particle spectra (see Table 2).

\section{FDS particles acceleration}

In a single energy spectrum fine dispersion structures look as one (electrons) or two (ions) narrow lines on the usual continuous background (see a typical ion spectrum in Lutsenko et al., 2005, Fig. 1). The full width at half maximum (FWHM) is $13-15 \%$ of the line energy. Obviously apart from the detector resolution the width must depend on the spectrum accumulation time $\Delta t$, the particles injection duration $\Delta T_{\text {inj }}$ and on the longitudinal dimension of the injection region $\Delta \varphi$. The analysis of all components of FWHM made in Lutsenko et al. (2005) showed that upper limits for the last two quantities are: $\Delta T_{\text {inj }}<95 \mathrm{~s}, \Delta \varphi<1.9 \mathrm{~h}$ MLT. Taking into account an unavoidable spread in trajectories by particles propagation from a distant acceleration point in the plasma sheet to a drift start position we can expect that the duration of the acceleration process and dimensions of the corresponding region must be very small.
On 22 December 1996 the Interball-1 spacecraft moved more than $6 \mathrm{~h}$ along the neutral sheet (13:20-19:20 UT). It was at normal, quiet states of the magnetosphere and solar wind: $K_{p}=23 \div 27, D_{s t}=-10 \div-30 \mathrm{nT}, P_{S W}=1.1 \div 1.3 \mathrm{nP}$, $V_{S W}=393 \div 417 \mathrm{~km} / \mathrm{s}, N p=4.1 \div 5.1 \mathrm{~cm}^{-3}, B_{\mathrm{YIMF}}=$ $0 \div-4 \mathrm{nT}, B_{\mathrm{ZIMF}}=-2 \div-5 \mathrm{nT}$ (IMF). Figure 14 shows magnetic field and energetic particle fluxes during the first hour of this interval. After 13:55 UT as the magnetic field as particle spectra become highly variable. Three short similar positive spikes in a $B_{Z}$-component (\#1, \#2 and \#3 in Fig. 15, left) with amplitudes $\sim 8 \mathrm{nT}$ are caused obviously by spontaneous current filament disruptions following by lobes magnetic field "reconnection" at the spacecraft positions $\left(X_{\mathrm{GSM}}=-23.08 R_{E}, Y_{\mathrm{GSM}}=12.05 R_{E}, Z_{\mathrm{GSM}}=-2.33 R_{E}\right)$. A zoomed part near the spike \#2 (see Fig. 14, right) shows that duration of this disruption was only $21 \mathrm{~s}$ after which the current filament was recovered. During the whole transition through the neutral sheet on 22 December 1996 more then 40 such spikes were observed.

Figure 15a illustrates a situation near disrupted current filament. Before the disruption the current sheet magnetic field 

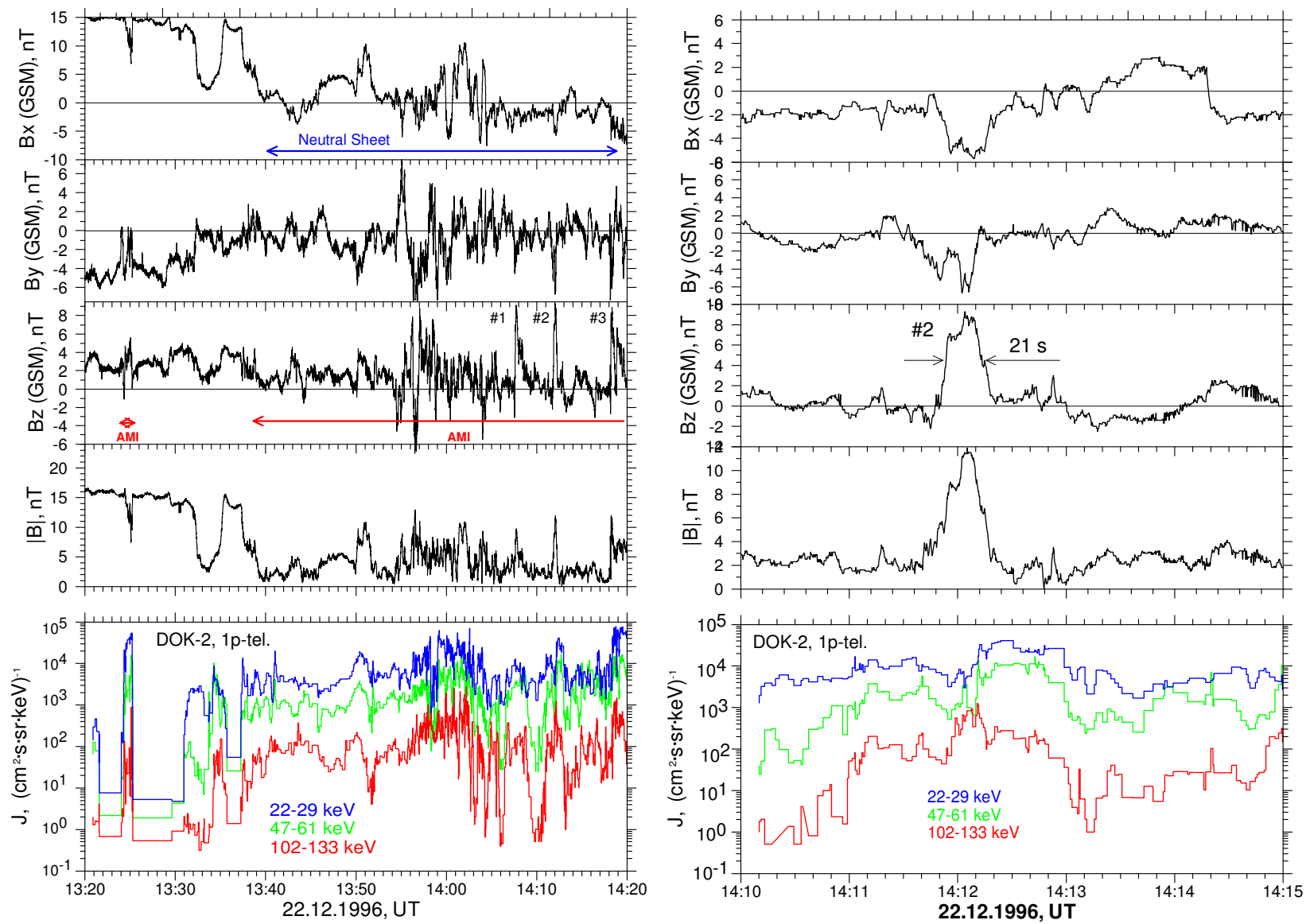

Fig. 14. Magnetic field and energetic particles observations in the neutral sheet by Interball-1. A zoomed part of graphs near $B_{Z}$ spike \#2 is shown left.

separates two geotail lobes and prevents their magnetic field shortening favorable from energetics considerations. After the disruption two types of electric field arise at about the same time:

1. a potential field $E_{\text {pot }}$ proportional to an electric circuit emf (with a possible addition of short spike of selfinductance field);

2. an inductive field $E_{\text {ind }}$ proportional to $\mathrm{dB}_{Z} / \mathrm{dt}$.

It is significant that the two types of electric fields occupy close but different regions. The first one is localized in a small disrupted part of the current filament, while the second one in a greater space around the potential electric field region. The $E_{\text {pot }}$ accelerate each plasma ions species to the energy $E=E_{\mathrm{pot}} \cdot d \cdot q$ creating Almost Monoenergetic Ions (Lutsenko, 2001). Here $d$ is dimension of the disruption region along the $E_{p o t}$ and $q$ is the ion charge. Our observations of ion spectra in the neutral sheet showed that lines with $E=50$ $150 \mathrm{keV}$ (FWHM 30\%) and 10-20 s duration permanently arise and disappear here. Figure 15b shows an example of such spectra having one almost monoenergetic ion line with
$E=79.4 \mathrm{keV}$ and $\mathrm{FWHM}=22.6 \mathrm{keV}$. In general case particles in the acceleration region experience an $\boldsymbol{E}_{\text {pot }} \times \boldsymbol{B}$ drift sweeping them from the region. To obtain the same full energy in the potential electric field all ions must reach the opposite side of the region what will be possible if the region width $d$ along the electric field is lower then ions gyroradii $R_{c}$ at full energy. For $80 \mathrm{keV}$ protons the $R_{c}=0.6 R_{E}$ by $B$ magnitude of $10 \mathrm{nT}$. So we find the upper estimate for $d$ : $d<0.6 R_{E}=3800 \mathrm{~km}$. Due to much smaller gyroradii electrons will be swept by the $\boldsymbol{E}_{\text {pot }} \times \boldsymbol{B}$ drift from the disruption region without acceleration and it explains why we never observed any lines in electron spectra here. It allows finding a lowermost estimate for the $d$ value. At $E=80 \mathrm{keV}$ and $B=10 \mathrm{nT}$ the electron gyroradius is $100 \mathrm{~km}$ and $d$ must be greater then this value. So acceleration of particles by a potential electric field allows making rough estimates for dimensions of corresponding region. It can be expected that dimensions of region where acceleration by the inductive electric field occurs must be several times greater $\left(1-2 R_{E}\right)$ but stay nevertheless much lower then characteristic dimensions of the plasma sheet. 
(a)

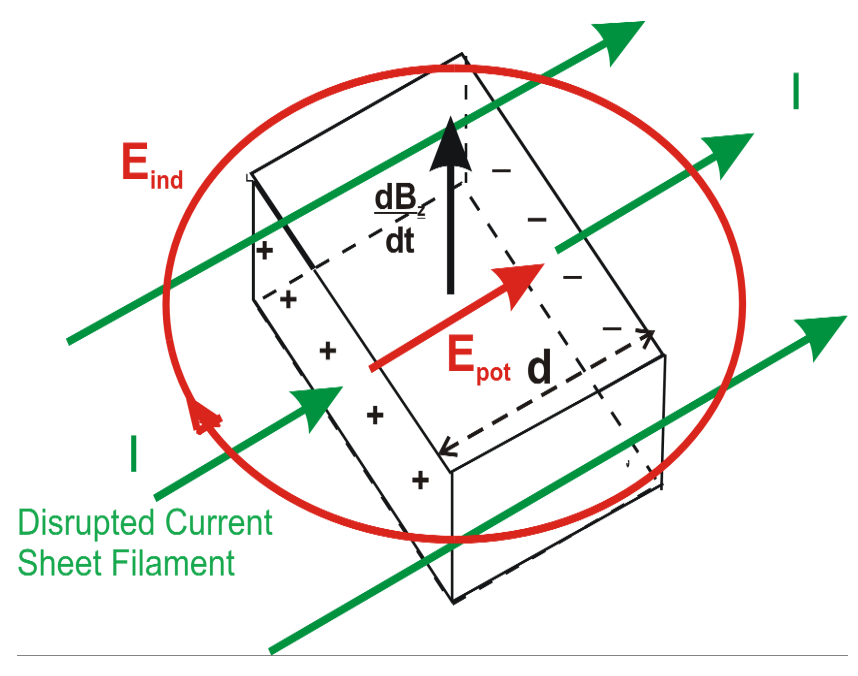

(b)

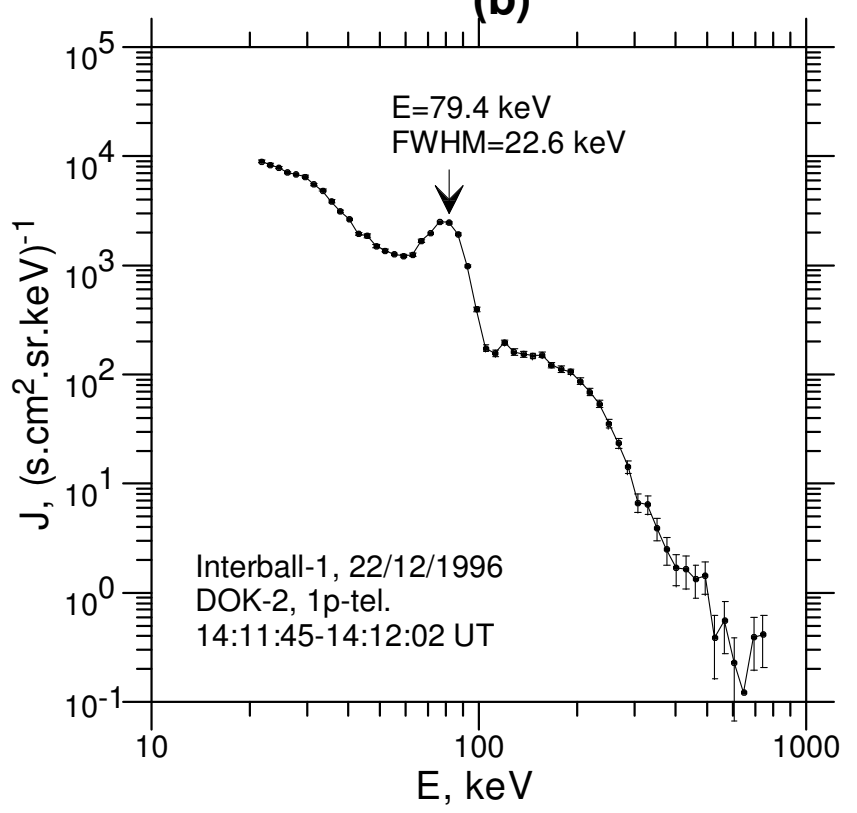

Fig. 15. (a) Electric fields at a current filament disruption. (b) One ion spectrum in the neutral sheet near the $B_{Z}$ spike \#2. A narrow peak with $E=79.4 \mathrm{keV}$ is a result of ion acceleration by the potential electric field, a broad bump at $E=100-400 \mathrm{keV}-$ possibly by the inductive electric field.

In contrast to the potential electric field the inductive one accelerates both ions and electrons and produce continuous particles spectra. The specific shapes of these spectra (power low for ions and exponential for electrons) were predicted theoretically and completely confirmed by our measurements of average spectra in the plasma sheet (Zelenyi et al., 1998; Taktakishvili et al., 1998). So this permanently working mechanism may be a main provider of energetic particles in the plasma sheet. A small part of these particles after propagation to the outer magnetosphere start drift around the Earth and form FDS. Possibly short time variations of the magnetic field in the near plasma sheet region allow these particles to be captured on drift shells. As the duration of the acceleration process is $<20 \mathrm{~s}$ and dimension $d<1-2 R_{E}$ this model explains small values of $\Delta T_{\mathrm{inj}}$ and $\Delta \varphi$ followed from FWHM of FDS lines analysis.

\section{Conclusions}

1. It was found that the FDS in energetic particle spectra in the outer magnetosphere are very ordinary phenomenon. They were not observed before Interball mission only because of insufficient energy and time resolutions of instruments used. On average at a quiet magnetosphere state one distinct FDS event of drift type was observed in $0.13 \mathrm{~h}$. The frequency of "time-of- flight" type FDS is much greater: $8-10$ per hour (by observations in one point).
2. Statistical data and particle motion simulations confirm our hypothesis on the FDS nature as a result of pulse injections of energetic particles to the night side of the outer magnetosphere followed by gradientcurvature drift around the Earth. As it was shown, only a small part $(\ll 1.4 \%)$ of all energetic particles permanently "bombarding" the outer magnetosphere from the plasma sheet can penetrate inside and start the drift around the Earth. This drift motion is rather stable and some particles can make at least 1-3 full turn before they escape. It takes from 0.5 to $2 \mathrm{~h}$ and in some cases the process can be observed from one spacecraft location.

3. Statistical analysis of FDS events observations showed that the disturbed states of the magnetosphere and the solar wind have no preferences in FDS particles generation.

4. Particles responsible for FDS have hard energy spectra. At high energies $(E>100 \mathrm{keV})$ intensities in FDS lines exceed those for a background spectrum more than 10 times. It was shown that such integral parameters as the total energy density and the total energy flux from a single injection can exceed 50-60\% of corresponding values for average ambient energetic particle spectrum in auroral regions. So FDS events particles can give an essential addition to the energetic particle population here to compensate for natural particle losses. 
5. The FDS particle acceleration can occur in the magnetotail plasma sheet (neutral sheet) by short pulses of an inductive electric field caused by spontaneous current filaments disruptions. The permanent acceleration of particles in the neutral sheet can replenish energetic particle population as in the plasma sheet itself as in the outer magnetosphere via FDS particles.

Acknowledgements. This work was supported by INTAS grant No 05-1000008-8050.

Topical Editor I. A. Daglis thanks two anonymous referees for their help in evaluating this paper.

\section{References}

Kudela, K., Lutsenko, V. N., Sibeck, D. G., Slivka, M., Grechko, T. V., and Sarris, E. T.: High energy particle dispersion events observed by Interball-1 and -2, Adv. Space Res., 30, 2849-2854, 2002.

Lutsenko, V. N., Kudela, K., and Sarris, E. T.: The DOK-2 Experiment to study of energetic particles by the tail and auroral probe satellites in the Interball project, Cosmic Res., 36(1), 98-107, 1998.

Lutsenko, V. N., Grechko, T. V., and Kudela, K.: Interball-1 and -2 observations of energy dispersion events in auroral zone for 30 $500 \mathrm{keV}$ ions and electrons, Proc. 5-th International Conference on Substorms, St. Petersburg, Russia, 16-20 May 2000, ESA SP443, 519-522, 2000.
Lutsenko, V. N.: Almost Monoenergetic Ions: New Support for Alfven Ideas on the Role of Electric Currents in Space Plasmas?, Phys. Chem. Earth, Part C, 26(8), 615-619, 2001.

Lutsenko, V. N., Gretchko, T. V., Kobelev, A. V., and Kudela, K.: Dispersion structures in the energetic ion and electron spectra in the auroral regions: their nature, properties and implication, Adv. Space Res., 30, 1787-1792, 2002a.

Lutsenko, V. N., Gretchko, T. V., Kobelev, A. V., Styazhkin, V. A., and Kudela, K.: "Wavy" energetic ion dispersion events and PC-5 type magnetic field pulsations, Adv. Space Res., 30, 17831786, $2002 b$.

Lutsenko, V. N., Kirpichev, I. P., Grechko, T. V., and Delcourt, D.: Source Positions of Energetic Particles Responsible for the Fine Dispersion Structures: Numerical Simulation Results, Planet. Space Sci., 53, 275-281, 2005.

Roederer, J. G.: Dynamics of geomagnetically trapped radiation, Springer-Verlag, Heidelberg - New York, 1970.

Sarafopoulos, D. V., Sidiropoulos, N. F., Sarris, E. T., Lutsenko, V. N., and Kudela, K.: The dawn-dusk asymmetry of energetic particles: an Interball perspective, J. Geophys. Res., 106, $13053-$ $13065,2001$.

Volland, H.: A Model of the Magnetospheric Electric Convection Field, J. Geophys. Res., 83, 2695-2699, 1978.

Zelenyi, L. M., Taktakishvili, A., Lutsenko, V. N., and Kudela, K.: Interball observations of the energetic particle spectra in the plasma sheet: indirect evidence of multiple explosive-like spontaneous reconnections, Substorms-4, edited by: Kokubun, S. and Kamide, Y., p. 521-526, 1998.

Taktakishvili, A. L., Zelenyi, L. M., Lutsenko, V. N., and Kudela, K.: On the Spectra of Energetic Particles in the Earth's Magnetotail, Cosmic Res., 36(3), 265-273, 1998. 